In Cres. Vol. $3 N^{\circ}$ 2: pp. 289-298, 2012

\title{
ESTILO DE VIDA Y FACTORES SOCIOCULTURALES DE LA ADULTA MAYOR DEL P.J. $1^{\circ}$ DE MAYO*
}

\author{
LIFE STYLE AND SOCIOCULTURAL FACTORS \\ OF THE ELDERLY WOMEN IN THE SHANTY TOWN \\ OF $1^{\circ} D E M A Y O$
}

Viviana Nohemi Yenque Aguilar ${ }^{1}$

\section{RESUMEN}

En el presente trabajo de investigación se planteó la siguiente hipótesis: existe relación estadísticamente significativa entre el estilo de vida y los factores socioculturales de la adulta mayor en el P.J. $1^{\circ}$ de Mayo -Nuevo Chimbote. La investigación fue de tipo cuantitativo, de diseño descriptivo correlacional. La muestra estuvo constituida por 117 adultas mayores, de una población de 166. Se encontró los siguientes resultados: la mayoría de las adultas mayores presentan un estilo de vida no saludable; sin embargo, existe un porcentaje significativo con estilo de vida saludable. En relación a los factores biosocioculturales se encontró que, en el factor cultural la mayoría presentan grado de instrucción primaria incompleta y profesan la religión católica. Así mismo, la mayoría son casadas, se ocupan como amas de casa y su ingresos económicos fluctúan entre 100 y 599 nuevos soles mensuales. Finalmente, aplicando la prueba estadística Chi-cuadrado de independencia con un nivel de significancia del $5 \%$, se comprobó que no existe relación estadísticamente significativa entre el estilo de vida y los factores socioculturales.

PALABRAS CLAVE: Estilo de vida, Factores socioculturales y Adulta mayor.

* Recibido: 04 de mayo del 2012; aprobado: 25 de octubre del 2012.

1 Licenciada en Enfermaría. Universidad Católica Los Ángeles de Chimbote. Enfermera Asistencial en el Policlínico San Luis - Chimbote. Realiza estudios en la especialidad de Centros Quirúrgicos en la Universidad Mayor de San Marcos. 


\section{ABSTRACT}

This nonexperimental research of descriptive corelational design aimed to establish the relationship between life style and sociocultural factors of the elderlywomen in the Shanty town of $1^{\circ}$ de Mayo, Nuevo Chimbote. at Javier Eraud shanty town-Santa. The sample consisted of 117 elderly women from a population of 166 . The data was statistically analyzed by means of the Chi Square test for independence with a $5 \%$ significance level. The study came to the following conclussions: There is no statistically significant relationship between lifestyle and sociocultural factors. Most of the elderlywomen present unhealthy lifestyle, however a significant number of them present healthy one. Respect to the biosociocultural factors, most have incompleted primary school level and are catholics. Also, most of them are married, work as a house wife, their salary range from 100 and 599 nuevos soles monthly.

KEY WORDS: Lifestyle, Sociocultural factors and Elderly women.

\section{INTRODUCCIÓN}

El presente trabajo de investigación se fundamenta en las bases conceptuales de estilo de vida y factores socioculturales de la adulta mayor, entendiendo que el estilo de vida es la forma de vida que se basa en patrones de comportamiento identificables, determinados por la interacción entre las características personales individuales, las interacciones sociales y las condiciones de vida socioeconómica y ambiental. Los factores socioculturales, supone una íntima unión y relación entre lo social y lo cultural, de tal manera que lo social sería el conjunto de relaciones que se establecen entre las personas de un grupo o territorio determinado. Y la cultura, por su parte, la podemos definir como el conjunto de valores, hábitos y costumbres. ${ }^{1,2}$

\section{OBJETIVO GENERAL}

Determinar la relación entre el estilo de vida y los factores socioculturales de la adulta mayor en el P.J. $1^{\circ}$ de Mayo - Nuevo Chimbote.

\section{OBJETIVOS ESPECÍFICOS}

a) Valorar el estilo de vida de la adulta mayor en el P.J. $1^{\circ}$ de Mayo Nuevo Chimbote.

b) Identificar los factores socioculturales: grado de instrucción, religión, estado civil, ocupación e ingreso económico de la adulta mayor en el P.J. $1^{\circ}$ de Mayo - Nuevo Chimbote.

c) Determinar si existe relación significativa entre las variables de estudio. 


\section{HIPÓTESIS}

Existe relación estadísticamente significativa entre el estilo de vida y los factores socioculturales de la adulta mayor en el P.J. $1^{\circ}$ de Mayo - Nuevo Chimbote.

\section{METODOLOGÍA}

La presente investigación fue de tipo cuantitativo, de corte transversal y diseño de investigación descriptivo-correlacional. La población estuvo conformada por 166 mujeres adultas mayores, que residen en el P.J. $1^{\circ}$ de Mayo del distrito de Nuevo Chimbote, del departamento de Áncash. Aplicando la técnica de muestreo obtuvimos una muestra de 117 mujeres adultas mayores, que residen en el P.J. $1^{\circ}$ de Mayo, del distrito de Nuevo Chimbote, del departamento de Áncash. Para recoger la información se utilizó dos instrumentos: la escala de estilo de vida y el cuestionario sobre factores biosocioculturales. Los datos fueron procesados en el paquete SPSS/info/software versión 18.0. Se presentaron los datos en tablas simples y se elaboraron gráficos para cada tabla. Para establecer la relación entre las variables de estudio se utilizó la prueba de independencia Chi cuadrado, con el 95\% de confiabilidad y significancia de $\mathrm{p}>0,05$.

\section{RESULTADOS}

Tabla 01

ESTILO DE VIDA DE LA ADULTA MAYOR. P. J. $1^{\circ}$ DE MAYO - NUEVO CHIMBOTE, 2011

\begin{tabular}{lcc}
\hline Estilo de vida & Frecuencia & Porcentaje \\
\hline Saludable & 14 & 12,0 \\
No saludable & 103 & 88,0 \\
\hline Total & 117 & 100,0 \\
\hline
\end{tabular}

Fuente: Escala de estilo de vida elaborada por: Walter, Sechrist y Pender, modificada por: Delgado R; Reyna E.; Díaz, R. Aplicado a la adulta mayor del P. J. 1ro. de Mayo, Enero 2011. 
Tabla 02

FACTORES SOCIOCULTURALES DE LA ADULTA MAYOR

\begin{tabular}{lrr}
\hline \multicolumn{3}{c}{ FACTORES CULTURALES } \\
\hline Grado de instrucción & $\mathbf{n}$ & $\%$ \\
\hline Analfabeta & 5 & 4,3 \\
Primaria incompleta & 97 & 82,9 \\
Primaria completa & 15 & 12,8 \\
Total & 117 & 100,0 \\
Religión & $\mathbf{n}$ & $\%$ \\
Católica & 70 & 59,8 \\
Evangélica & 36 & 30,8 \\
Otras & 11 & 9,4 \\
Total & 117 & 100,0 \\
\hline
\end{tabular}

FACTORES SOCIALES

\begin{tabular}{lrr}
\hline Estado civil & $\mathbf{n}$ & $\%$ \\
Soltera & 5 & 4,3 \\
Casada & 51 & 43,6 \\
Viuda & 48 & 41,0 \\
Unión libre & 6 & 5,1 \\
Separada & 7 & 6,0 \\
Total & 117 & 100,0 \\
Ocupación & $\mathbf{n}$ & $\mathbf{\%}$ \\
Obrera & 7 & 6,0 \\
Ama de casa & 86 & 73,5 \\
Ambulante & 23 & 19,6 \\
Otras & 1 & 0,0 \\
Total & 117 & 100,0 \\
Ingreso económico (soles) & $\mathbf{n}$ & $\mathbf{\%}$ \\
Menos de 100 & 41 & 35,0 \\
De 100 a 599 & 74 & 63,2 \\
De 600 a 1000 & 2 & 1,8 \\
Total & 117 & 100,0 \\
\hline
\end{tabular}

Fuente: Cuestionario de factores biosocioculturales, elaborado por: Delgado R; Reyna, E; Díaz, R. Aplicado a la adulta mayor del P. J. 1 de Mayo, Enero 2011. 
Tabla 03

FACTORES CULTURALES Y ESTILO DE VIDA DE LA ADULTA MAYOR

\begin{tabular}{|c|c|c|c|c|c|c|}
\hline \multirow[t]{3}{*}{$\begin{array}{l}\text { Grado de } \\
\text { instrucción }\end{array}$} & \multirow{2}{*}{$\begin{array}{c}\begin{array}{c}\text { Estilo de } \\
\text { vida }\end{array} \\
\text { Saludable }\end{array}$} & \multirow{2}{*}{$\begin{array}{c}\text { Total } \\
\text { No saludable }\end{array}$} & \multicolumn{4}{|c|}{$\begin{array}{c}\quad \mathrm{X}^{2}=0,369,2 \mathrm{gl} \\
\mathrm{P}=0,831>0,05 \\
\text { No existe relación estadísticamente } \\
\text { significativa. }\end{array}$} \\
\hline & & & & & & \\
\hline & $\mathrm{n}$ & $\%$ & $\mathbf{n}$ & $\%$ & $\mathbf{n}$ & $\%$ \\
\hline Analfabeta & 1 & 0,9 & 4 & 3,4 & 5 & 4,3 \\
\hline $\begin{array}{l}\text { Primaria } \\
\text { incompleta }\end{array}$ & 11 & 9,4 & 86 & 73,5 & 97 & 82,9 \\
\hline $\begin{array}{l}\text { Primaria } \\
\text { completa }\end{array}$ & 2 & 1,7 & 13 & 11,1 & 15 & 12,8 \\
\hline Total & 14 & 12,0 & 103 & 88,0 & 117 & 100,0 \\
\hline \multirow[t]{3}{*}{ Religión } & $\begin{array}{l}\text { Estilo de } \\
\text { vida }\end{array}$ & Total & \multicolumn{4}{|c|}{$\begin{array}{c}\qquad \mathrm{X}^{2}=0,895,2 \mathrm{gl} \\
\mathrm{P}=0,639>0,05 \\
\text { No existe relación estadísticamente } \\
\text { significativa. }\end{array}$} \\
\hline & Saludable & No saludable & & & & \\
\hline & $\mathrm{n}$ & $\%$ & $\mathbf{n}$ & $\%$ & $\mathbf{n}$ & $\%$ \\
\hline Católica & 10 & 8,5 & 60 & 51,3 & 70 & 59,8 \\
\hline Evangélica & 3 & 2,6 & 33 & 28,2 & 36 & 30,8 \\
\hline Otras & 1 & 0,9 & 10 & 8,5 & 11 & 9,4 \\
\hline Total & 14 & 12,0 & 103 & 88,0 & 117 & 100,0 \\
\hline
\end{tabular}

Fuente: Cuestionario de factores biosocioculturales, elaborado por: Delgado R; Reyna, E; Díaz, R. y escala de estilo de vida modificada por Delgado R; Reyna, E; Díaz, R. Aplicado a la adulta mayor del P. J. $1^{\circ}$ de Mayo, Enero 2011. 
Tabla 04

FACTORES SOCIALES Y ESTILO DE VIDA DE LA ADULTA MAYOR

\begin{tabular}{|c|c|c|c|c|c|c|}
\hline \multirow[t]{3}{*}{$\begin{array}{c}\text { Estado } \\
\text { civil }\end{array}$} & \multirow{2}{*}{$\begin{array}{c}\begin{array}{c}\text { Estilo de } \\
\text { vida }\end{array} \\
\text { Saludable }\end{array}$} & Total & \multicolumn{4}{|c|}{$\begin{array}{l}\qquad \mathrm{X}^{2}=6,853,4 \mathrm{gl} \\
\mathrm{P}=0,144>0,05 \\
\text { No existe relación estadísticamente significativa. }\end{array}$} \\
\hline & & No saludable & & & & \\
\hline & $\mathrm{n}$ & $\%$ & $\mathbf{n}$ & $\%$ & $\mathbf{n}$ & $\%$ \\
\hline Soltera & 0 & 0,6 & 5 & 4,3 & 5 & 4,3 \\
\hline Casada & 4 & 3,4 & 47 & 40,2 & 51 & 43,6 \\
\hline Viuda & 10 & 8,5 & 38 & 32,5 & 48 & 41,0 \\
\hline Unión libre & 0 & 0,0 & 6 & 5,1 & 6 & 5,1 \\
\hline Separada & 0 & 0,0 & 7 & 6,0 & 7 & 6,0 \\
\hline Total & 14 & 12,0 & 103 & 88,0 & 117 & 100,0 \\
\hline \multirow[t]{3}{*}{ Ocupación } & $\begin{array}{l}\text { Estilo de } \\
\text { vida }\end{array}$ & Total & \multicolumn{4}{|c|}{$\begin{array}{c}\mathrm{X}^{2}=2,178,3 \mathrm{gl} \\
\mathrm{P}=0,536>0,05 \\
\text { ación estadísticamente }\end{array}$} \\
\hline & Saludable & No saludable & & & & \\
\hline & $\mathrm{n}$ & $\%$ & $\mathbf{n}$ & $\%$ & $\mathbf{n}$ & $\%$ \\
\hline Obrera & 2 & 1,7 & 5 & 4,3 & 7 & 6,0 \\
\hline Ama de casa & 9 & 7,7 & 77 & 65,8 & 86 & 73,5 \\
\hline Ambulante & 3 & 2,6 & 20 & 17,1 & 23 & 19,7 \\
\hline Otros & 0 & 0,0 & 1 & 0,9 & 1 & 0,9 \\
\hline Total & 14 & 12,0 & 103 & 88,0 & 117 & 100,0 \\
\hline \multirow[t]{3}{*}{$\begin{array}{l}\text { Ingreso } \\
\text { económico } \\
\text { (soles) }\end{array}$} & $\begin{array}{l}\text { Estilo de } \\
\text { vida }\end{array}$ & Total & \multicolumn{4}{|c|}{$\begin{array}{l}\quad \mathrm{X}^{2}=0,277,2 \mathrm{gl} \\
\mathrm{P}=0,871>0,05\end{array}$} \\
\hline & Saludable & No saludable & & & & \\
\hline & $\mathrm{n}$ & $\%$ & $\mathbf{n}$ & $\%$ & $\mathbf{n}$ & $\%$ \\
\hline Menos de 100 & 5 & 4,3 & 36 & 30,8 & 41 & 35,0 \\
\hline $100-599$ & 9 & 7,7 & 65 & 55,6 & 74 & 63,2 \\
\hline $600-1000$ & 0 & 0,0 & 2 & 1,7 & 2 & 1,7 \\
\hline Total & 14 & 12,0 & 103 & 88,0 & 117 & 100,0 \\
\hline
\end{tabular}

Fuente: Cuestionario de factores biosocioculturales, elaborado por: Delgado R; Reyna, E; Díaz, R. y escala de

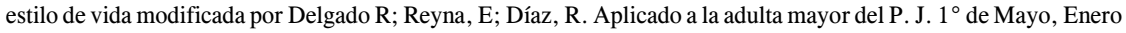
2011. 


\section{DISCUSIÓN}

Tabla 01: Se muestra que del 100\% (117) de adultas mayores que participaron en el estudio, el $12 \%$ (14) tienen un estilo de vida saludable y un $88 \%$ (103) no saludable.

Los resultados obtenidos evidencian que la mayoría de las adultas mayores del P.J. $1^{\circ}$ de Mayo, presentan un alto porcentaje de estilos de vida no saludables, lo cual indica que las mujeres adultas mayores no están adoptando conductas promotoras de salud, así como hábitos de alimentación saludables, descanso adecuado, actividad y ejercicio en forma periódica, tampoco asisten asisten una vez al año al establecimiento de salud. La población adulta mayor femenina se dedica solo al hogar; además, presentan bajo nivel cultural, factores que influyen en una adecuada alimentación. Este desconocimiento condiciona hábitos y modos de vida no saludables, indirectamente, produciéndoles complicaciones en su salud y vida diaria. Además, otro factor que influye es la edad muy avanzada de las encuestadas. Eso justifica por qué no realizan en forma regular actividad y ejercicio físico.

Los resultados antes mencionados son similares a los obtenidos por Carrasco, V. ${ }^{3}$, en su estudio sobre el Estilo de Vida y Estado Nutricional del Adulto Mayor en el A.H. Tres Estrellas, en Chimbote, en donde reporta que el $12,5 \%$ de adultos mayores presentan un estilo de vida saludable, mientras que el $87,5 \%$ presentan un estilo de vida no saludable.

Tabla 02: En la presente investigación se muestra la distribución porcentual de los factores socioculturales de las personas adultas mayores que residen en el P.J. $1^{\circ}$ de Mayo. En los factores culturales se observa que el mayor porcentaje de personas adultas mayores, en un $82 \%$ (96) tienen como grado de instrucción primaria incompleta; en cuanto a la religión, el 59,8\% (70) son católicos. En lo referente a los factores sociales, en el 43,6\% (51) su estado civil es casado, el 73,5\% (86) son amas de casa y el 63,2\% (74) tiene un ingreso económico entre 100 a 599 nuevos soles.

El P.J. $1^{\circ}$ de Mayo es una comunidad antigua. Debido a ello la gran cantidad de adultas mayores de avanzada edad, entre las investigadas existen porcentajes elevados de grado de instrucción primaria incompleta, debido a que anteriormente las familias priorizaban la educación para los varones y las mujeres no estudiaban o simplemente eran enviadas a los centros educativos para que aprendan a leer y escribir, porque la labor de la mujer solo se limitaba al cuidado del hogar y de los hijos, negándosele la oportunidad de superarse. Por ende, si no tienen un alto nivel de instrucción, no podrán autocuidarse y su estilo de vida no será saludable. 
Los resultados encontrados en la presente investigación difieren en algunos indicadores a los reportados por el Instituto Nacional de Estadística e Informática (INEI) ${ }^{4}$. Según el Censo Nacional 2007, en relación a los factores culturales, difieren porque el mayor porcentaje de adultos mayores presenta un grado de instrucción primaria, con un $41 \%$, y a nivel distrital de Chimbote, esta suma aumenta a un $46,33 \%$. En relación a la religión, los resultados son similares a los encontrados por nuestro estudio, ya que el INEI reporta a nivel nacional que el $82 \%$ de adultos mayores son católicos y a nivel del distrito de Chimbote, $74 \%$ son de religión católica.

En relación a los factores sociales, el INEI reporta que el 56\% de adultos mayores son casados, lo cual difiere con lo encontrado en nuestra investigación. En cuanto a la ocupación, los resultados se aproximan a los reportados por el INEI a nivel nacional. El $73 \%$ de los adultos mayores es una población económicamente no activa, siendo el 56\% que se dedica solo al cuidado del hogar y el $27 \%$ es una población económicamente activa. En el distrito de Chimbote, el $78 \%$ de los adultos mayores es una población económicamente no activa, siendo el $52 \%$ que se dedica solo al cuidado del hogar y el $22 \%$ es una población económicamente activa. ${ }^{4}$

Por los resultados obtenidos, se puede afirmar que cada persona adulta mayor en estudio tiene sus propias características socioculturales, las cuales van a influir en el comportamiento que cada persona ponga en práctica en su vida cotidiana, generando conductas favorables o desfavorables para su salud.

Tabla 03: con respecto a la relación entre los factores culturales y el estilo de vida, se observa que el 73,5\% (86) de los que pertenecen a un grado de instrucción primaria incompleta, tienen estilo de vida no saludable; seguido del 9,4\% (11), que también son de primaria incompleta y tienen estilo de vida no saludable. Por otro lado, se observa que el 51,3\% (60), que son católicos(as), tienen estilo de vida no saludable; mientras que un $8,5 \%$ (10), que también son católicos(as), tienen estilo de vida saludable.

Al relacionar el grado de instrucción con el estilo de vida utilizando la prueba estadística Chi-cuadrado de independencia $\left(\mathrm{X}^{2}=0,334,2 \mathrm{gl}\right.$ y $\left.\mathrm{p}>0,05\right)$, encontramos que no existe relación. Así mismo, haciendo uso del estadístico Chi-cuadrado de independencia $\left(\mathrm{X}^{2}=0,895,2 \mathrm{gl} \mathrm{y} \mathrm{p}>0,05\right)$, se encontró que no existe relación estadísticamente significativa entre la religión y el estilo de vida.

$\mathrm{Al}$ relacionar los factores culturales y el estilo de vida, encontramos que no existe relación entre ellos y cada variable es independiente de la otra; las adultas 
mayores con un grado de instrucción primaria incompleta presentan altos porcentajes de estilo de vida no saludable y porcentajes significativos con estilo de vida no saludable.

Estos resultados coinciden con los obtenidos por Pujay ${ }^{5}$ en su estudio Estilo de Vida y Factores Biosocioculturales del adulto mayor en la Clínica Geriátrica de San Isidro Labrador en Ate-Lima, quien reportó que no existe relación estadísticamente significativa entre estilo de vida y los factores culturales.

Tabla 04: con respecto a la relación entre los factores sociales con el estilo de vida, se observa que el 40,2\% (30), que son casadas, tienen estilo de vida saludable; seguido del 3,4\% (4), que son casadas, tienen estilo de vida no saludable. Por otro lado, se observa que un 65,8\% (77) pertenece a la ocupación amas de casa y tienen estilo de vida no saludable; seguido de un $17,1 \%$ (20) que son ambulantes y tienen estilo de vida no saludable. Por último, se muestra que el 55,6\% (65), con ingreso económico entre 100 a 599 soles mensuales, tienen estilo de vida no saludable; seguido del 30,8\% (36) que, aunque también cuentan con 100 a 599 soles de ingreso económico, tienen estilo de vida saludable.

$\mathrm{Al}$ relacionar el estado civil con el estilo de vida, utilizando la prueba estadística Chi-cuadrado de independencia $\left(X^{2}=6,853,4 \mathrm{gl}\right.$ y $\left.\mathrm{p}>0,05\right)$, encontramos que no existe relación estadísticamente significativa entre ambas variables. Asimismo, según la prueba estadística Chi-cuadrado de independencia $\left(\mathrm{X}^{2}=2,178,3 \mathrm{gl} \mathrm{y} \mathrm{p}>0,05\right)$, se encontró que no existe relación estadísticamente significativa entre las variables. También la prueba estadística Chi-cuadrado de independencia $\left(\mathrm{X}^{2}=0,277,2 \mathrm{gl}\right.$ y $\left.\mathrm{p}>0,05\right)$ se encontró que no existe relación estadísticamente significativa entre el ingreso económico y el estilo de vida.

Lo encontrado coincide con lo reportado por Felipe, L. ${ }^{6}$ en su estudio Estilo de Vida y Factores Biosocioculturales de la persona adulta mayor, A.H. Villa Magisterial I y II Etapa, Nuevo Chimbote, 2010, quien concluye que no existe relación estadísticamente significativa entre el estilo de vida y factores sociales.

\section{CONCLUSIONES}

1a. La mayoría de las adultas mayores presentan un estilo de vida no saludable y un porcentaje significativo un estilo de vida saludable.

2a. Respecto a los factores socioculturales de las adultas mayores en el P.J. $1^{\circ}$ de Mayo tenemos: en el factor cultural, la mayoría presentan grado de instrucción primaria incompleta y más de la mitad profesan la religión católica. 
3a. En el factor social, menos de la mitad, su estado civil es casada, la mayoría es ama de casa y más de la mitad tienen ingresos económicos entre 100 y 599 nuevos soles mensuales.

4a. No existe relación estadísticamente significativa $(p>0,05)$ entre el estilo de vida y los factores socioculturales: grado de instrucción, estado civil, religión, e ingreso económico de las adultas mayores en el P.J. $1^{\circ}$ de Mayo Nuevo Chimbote.

\section{REFERENCIAS BIBLIOGRÁFICAS}

1 Soto, L. Estilos de Vida y Calidad de Vida. [Serie Internet] [Citado el 2011 Febrero 04] [Alrededor de 1 planilla]. Disponible desde el URL: http://www.mitecnologico.com/Main/ EstilosDeVidaYCalidadDeVida.

2 Moguer M. Sociocultural. [Serie Internet] [Citado el 2011 Febrero 04] [Alrededor de 1 planilla]. Disponible desde el URL: http://mjomog7.blogspot.com/2007/10/definicin-desociocultural.html.

3 CARRASCo V. Estilo de Vida y estado nutricional del adulto mayor en el A. H. Tres estrellas de Chimbote. [Tesis para optar el título de licenciado en enfermería]. Chimbote: Universidad Católica los Ángeles de Chimbote; 2010.

4 InSTITUto NACIONAL DE ESTADístiCA E InFORMÁTICA. Censos Nacionales 2007. [Serie Internet] [Citado el 2011 Enero 20] [Alrededor de 4 planillas]. Disponible desde el URL: http://desa.inei. gob.pe/censos2007/tabulados/.

5 Pujay, Y. Estilo de vida y factores biososioculturales del adulto mayor, clínica geriátrica san Isidro labrador ATE. [Tesis para optar el título de licenciada en enfermería]. Lima: Universidad Católica los Ángeles de Chimbote; 2009.

6 FELIPE L. Estilo de Vida y factores biosocioculturales del adulto mayor del A.H Villa Magisterial I y II etapa. [Tesis para optar el título de licenciada en enfermería]. Chimbote: Universidad Católica los Ángeles de Chimbote; 2010. 